\title{
Polymeric Liquid Templating of Hierarchical Porous Films by Nanofibrillar Alginic Acid Assemblies
}

\author{
Thai-Hoa Tran ${ }^{1}$, Sy-Thang Ho ${ }^{2}$, Thanh-Dinh Nguyen ${ }^{3 *}$ \\ ${ }^{1}$ Department of Chemistry, College of Sciences, Hue University, Hue, Vietnam; ${ }^{2}$ Department of Chemistry, Dong Thap University, \\ Dong Thap, Vietnam; ${ }^{3}$ Department of Chemical Engineering, Laval University, Quebec, Canada. \\ Email: *thanh-dinh.nguyen.1@ulaval.ca
}

Received July $9^{\text {th }}, 2013$; revised August $9^{\text {th }}, 2013$; accepted August $16^{\text {th }}, 2013$

Copyright (C) 2014 Thai-Hoa Tran et al. This is an open access article distributed under the Creative Commons Attribution License, which permits unrestricted use, distribution, and reproduction in any medium, provided the original work is properly cited. In accordance of the Creative Commons Attribution License all Copyrights (C) 2014 are reserved for SCIRP and the owner of the intellectual property Thai-Hoa Tran et al. All Copyright (C) 2014 are guarded by law and by SCIRP as a guardian.

\section{ABSTRACT}

Hierarchical phases of the biomaterials can be used as template to transfer their intricate organization into biomimic inorganic solids. Herein, hierarchical mesoporous silica films with aligned pores have been templated by nanofibrillar alginic acid. An aqueous suspension of the alginic acid nanofibers was prepared by treating the brown seaweeds with sodium carbonate solution and subsequent precipitation in dilute hydrochloric acid. The alginic acid nanofibers of the organization into a hierarchical aligned phase in an acetic acid-sodium acetate buffer were used to template silica-alginic acid composite films by evaporation-induced self-assembly of alkoxysilane with nanofibrillar alginic acid. Calcination of the alginic acid template afforded hierarchical mesoporous silica glasses. Carbonization of the silica-alginic acid composites and subsequent etching the silica recovered mesoporous carbon supercapacitors.

\section{KEYWORDS}

\section{Nanofibrillar Alginic Acid; Hierarchial Organization; Mesoporosity; Silica Films; Carbon Supercapacitors}

\section{Introduction}

Sophisticated biomaterials are normally fabricated in nature by oriented hierarchical organization of simple nanoscale elements [1,2]. Polymeric liquid templating offers a biomimetic approach to synthesizing materials with intricate organization that mimic the sophisticated structures found in nature $[3,4]$ The use of the polymeric liquid templating could allow to control the order of the morphology and structural orientation of such materials [5-7]. Mesoporous silica materials synthesized via polymeric liquid templating with a surfactant have been the subject of widespread research in materials chemistry $[8,9]$. The mesochannel structured materials have unique optical properties, giving them potential opportunities in applications as optical elements [10], catalyst supports [11], adsorbents [12], and solar cells [13].

Hierarchical phases of the biomacromolecules of polysaccharides, collagen, proteins, and DNA can be use as

*Corresponding author. biotemplates to prepare solid-state materials [1,14]. Alginic acid, a linear copolymer consisting mainly of residues of $\beta$-1,4-linked D-mannuronic acid and $\alpha$-1, 4-linked L-glucuronic acid, is the main structural component of the cell walls of brown seaweeds $[15,16]$. Alginic acid, which is found in the brown seaweeds, is an abundant polysaccharide in the ocean. Alginic acid extracted from the seaweeds could be used as a renewable resource for tissue engineering as delivery vehicle for drugs and gelling agent for food products.

Alginic acid fibers are typically prepared by treating the brown seaweeds with alkaline conditions and subsequent precipitation in dilute hydrochloric acid [16]. Aqueous suspensions of the alginic acid could be stabilized in a buffer solution. The exposed carboxylic acid groups on the polymeric chains could be partially deprotonated to provide negative surface charge, which leads to repulsive forces between fibers. The extent of the deprotonation changes the surface charge density and could affect the critical concentration for the formation of the hierarchical 
organization of the alginic acid fibers. By slowly drying the aqueous alginic acid suspension might give organic films with hierarchical organization.

The hierarchical phase of the nanofibrillar alginic acid (NAA) could potentially be used as a template to create porous solid-state materials with hierarchical structure and high surface area. Recent efforts have used polysaccharides to template the mesoporous silica solids. The pioneering work of Manner et al. [17] reported the first demonstration of chiral nematic mesoporous silica materials through nanocellulose template-directed condensation of tetraethoxysilane precursor. Alonso et al. $[18,19]$ demonstrated the liquid templating of aligned mesochannel silica materials by nanochitin assemblies. In other papers, Mann [20] and Brunner [21] also demonstrated $\beta$-chitin-templated mineralization of chitin-silica composites. These novel materials typically possess supramolecular organizations of the structures and morphologies transcribed from the hierarchical template.

Mesoporous carbon materials have a potential use in capacitive energy storage and conversion [22]. One of the most critical aspects in the development of the supercapacitors is thus to extend their rechargeable capacity. The materials of carbon nanotubes, graphene, and graphene oxide have showed high electric doublelayer capacitance performances $[23,24]$. Confining appropriate metal oxide particles into porous carbon support could improve the electrochemical energy storage through pseudo-capacitive redox reactions [25]. Synthesis of the porous carbon materials using natural-based polymers as a renewable carbon resource offers in terms of economic advantages and potential applications [26]. Recent efforts have been made to carbonize alginic acid to porous carbon supercapacitors with superior electrical conductivity. The pioneering work of Raymundo-Pinero and coworkers [15] demonstrated the first examples of pore carbon formed via direct pyrolysis of the alginic acid-contained seaweeds. Later studies on pure alginic acid showed that it is carbonized to porous carbon [27].

The nanofibrillar alginic acid generally formed disordered hydrogel structures from hydrogen bonding. Alginic acid is a biopolymer carrying more carboxyl groups and tends to form hydrogels and swells in water. Thus, it is often difficult to cast a solid film without using crosslinkers. We hypothesized that whether aqueous alginic acid suspensions could be prepared and dried to give self-assembled solid films. By combining the sol-gel and self-assembly chemistry in this alternative would provide new materials in a film form with hierarchical porous structures.

Herein, we present the first examples of the polymeric liquid templating of hierarchical porous films by nanofibrillar alginic acid assemblies. Hierarchical alginic acid fibers extracted from the brown seaweeds were used to template silica-alginic acid composites upon evaporation-induced self-assembly. Subsequent removal of the alginic acid template in the composites by heating generated mesoporous silica films with hierachical organization, high specific surface area, and optical anisotropy. The carbonization and subsequent etching of silica-NAA composites recovered hierarchical mesoporous carbon films that function as an electrical supercapacitor with highly electrical capacitance.

\section{Experimental Section}

Starting materials: Brown seaweeds (Sargassum turbinarioides) were collected in LangCo beach, Thua ThienHue province, Vietnam in 2011. The seaweeds were washed abundantly with water and dried at $60^{\circ} \mathrm{C}$ for 3 days prior to lab use. Tetramethyl orthosilicate $(99 \%)$ was purchased from Sigma-Aldrich. Other chemicals of sodium carbonate, hydrochloric acid, formaldehyde, ethanol, sodium hydroxide, acetic acid, sodium acetate, and ammonia were obtained from standard suppliers.

Preparation of NAA films from brown seaweeds: 20 grams of the dried seaweeds were soaked in $800 \mathrm{~mL}$ formaldehyde 2 vol.\% at room temperature for $24 \mathrm{~h}$ to remove phenolic compounds. Next, the seaweeds were washed three times with $1000 \mathrm{~mL}$ water and added to $800 \mathrm{~mL} \mathrm{HCl} 0.2 \mathrm{M}$ for $24 \mathrm{~h}$ to soluble mineral salts. The samples were rewashed with water and then added to 800 $\mathrm{mL} \mathrm{Na}_{2} \mathrm{CO}_{3} 2 \%$ and heated at $95^{\circ} \mathrm{C}$ for $3 \mathrm{~h}$ for alginate extraction. The soluble fraction was collected by filtration and sodium alginate was precipitated by three volumes of ethanol $95 \%$. Sodium alginate collected was washed twice by $100 \mathrm{~mL}$ of acetone and dissolved in 50 $\mathrm{mL}$ water. Alginic acid gels were obtained by adding 50 $\mathrm{mL} \mathrm{HCl} 0.2 \mathrm{M}$ to the aqueous sodium alginate solution. The gels were filtered and redispersed in water. The yield of the alginic acid extraction from the brown seaweeds was ca. $10 \mathrm{wt} \%$. The $\mathrm{pH}$ of the gel solution was adjusted to 4.5 using $0.1 \mathrm{M}$ acetic acid and $0.1 \mathrm{M}$ sodium acetate buffer and allowed to form an aqueous alginic acid suspension with the concentration of $5.0 \mathrm{wt} \%$. To increase the stabilization of alginic acid, the aqueous suspension was sonicated for $1 \mathrm{~h}$ using an ultrasonic instrument (50 $\mathrm{Hz}, 120 \mathrm{~W}$ ). For the preparation of pure NAA films, 10 $\mathrm{mL}$ of the aqueous NAA suspension (5.0 wt\%, $\mathrm{pH} 4.5)$ was poured to a $60 \mathrm{~mm}$-sized polystyrene Petri dish and allowed to evaporate at ambient conditions to form a NAA film after $72 \mathrm{~h}$. The films were soaked in anhydrous ethanol for $15 \mathrm{~min}$ to remove water through a solvent exchange process. The crack-free solid films were obtained after vacuum-drying at room temperature overnight.

Preparation of mesoporous silica films: A specified amount (543 mg) of tetramethyl orthosilicate was mixed to $10 \mathrm{~mL}$ of the aqueous NAA suspension $(5.0 \mathrm{wt} \%, \mathrm{pH}$ 
4.5) with silica/NAA weight ratio of 30:70 wt\% under stirring at room temperature for $1 \mathrm{~h}$ to form a silica-NAA composite suspension. After hydrolysis of tetramethyl orthosilicate, $0.6 \mathrm{~mL}$ of concentrated ammonia was added to the composite suspension to reduce hydrogen bonds of alginic acid. The composite suspension was then poured to a $60 \mathrm{~mm}$-sized polystyrene Petri dish and allowed the evaporation-induced self-assembly at ambient conditions to form a silica-NAA composite film after 72 $\mathrm{h}$. The composite film was soaked in anhydrous ethanol for $15 \mathrm{~min}$ to remove water, and the films became more hardness after evaporating ethanol. Vacuum-drying at room temperature overnight yielded a crack-free composite solid film.

To form mesoporous silica films, the NAA-silica composite films were pyrolyzed under air at a heating rate of $5^{\circ} \mathrm{C} \cdot \mathrm{min}^{-1}$ to $100^{\circ} \mathrm{C}$, held at $100^{\circ} \mathrm{C}$ for $4 \mathrm{~h}$, then heated to $550^{\circ} \mathrm{C}$ at $5^{\circ} \mathrm{C} \cdot \mathrm{min}^{-1}$, and held at $540^{\circ} \mathrm{C}$ for $6 \mathrm{~h}$. After slowly cooling the sample to room temperature, silica films were obtained $(90 \mathrm{mg}$ of the silica films was obtained from $500 \mathrm{mg}$ NAA-silica composites).

To gain the porous carbon films, the silica-NAA composite films were carbonized under flowing nitrogen at $100^{\circ} \mathrm{C}$ for $4 \mathrm{~h}$ and then heated to $200^{\circ} \mathrm{C}$ for $1 \mathrm{~h}$ and to $1000^{\circ} \mathrm{C}$ for $6 \mathrm{~h}$ with a heating rate of $1^{\circ} \mathrm{C} \mathrm{min}{ }^{-1}$ to reproduce silica-carbon composites. For removal of the silica component, the silica-carbon composites were heated in $200 \mathrm{~mL} \mathrm{NaOH} 2 \mathrm{M}$ at $90^{\circ} \mathrm{C}$ for $4 \mathrm{~h}$ to generate mesoporous carbon films. $62 \mathrm{~g}$ carbon films were obtained from $400 \mathrm{~g}$ silica-NAA composites.

\section{Characterization}

Powder X-ray diffraction (PXRD) pattern of the sample was recorded on an Advance Bruker D8 X-ray diffracttometer, using $\mathrm{Cu} \mathrm{K} \alpha$ radiation $(\lambda=1.5418 \AA)$. Raman spectrum was recorded on a Micro Raman LabRAM-1B spectrometer with $785 \mathrm{~nm}$ excitation. Scanning electron microscope (SEM) images of the samples were obtained on a Hitachi S4700 electron microscope. Breaking the films into small pieces placed on aluminum stubs and then coated with Au for silica and Au-Pd for carbon. Polarized optical microscopy (POM) was performed on an Olympus microscope. Transmission electron microscope (TEM) images and energy dispersive X-ray spectroscopy (EDX) analysis of the samples were obtained on a JEOLJEM 1010 microscopy. The nanofibrillar alginic acid sample was prepared by placing one drop $(0.1 \mathrm{~mL})$ of the dilute NAA solution (0.05 wt\%) onto a TEM grid after sonicating for $30 \mathrm{~min}$. To image the carbon films, the samples were gently ground to a fine powder, suspended in ethanol, then deposited onto a TEM grid. Thermogravimetric analysis (TGA) of the the carbon films ( 1 mg) was conducted at a heating rate of $5^{\circ} \mathrm{C} \cdot \mathrm{min}^{-1}$ under air to $900^{\circ} \mathrm{C}$ using a Labsys TG/DSCSE-TARAM thermogra- vimetric analyser. Fourier transform infrared absorption spectrum (FTIR) was recorded on a PERKIN ELMER FT-IR Spectrometer with the $\mathrm{KBr}$ pellet technique. Nitrogen adsorptiondesorption isotherms (US) were obtained using a Micromeritics at $77 \mathrm{~K}$ and the carbon film samples $\left(100 \mathrm{mg}\right.$ ) were degassed at $150^{\circ} \mathrm{C}$ in vacuum for $4 \mathrm{~h}$ efore measurements.

Cyclic voltammetry (CV) and galvanostatic charge/ discharge curves of the mesoporous carbon materials were recorded between 5 and $500 \mathrm{mV}$ using a CHI 760D potentiostat galvanostat (CH Instruments Inc.) with twoelectrode configuration. The dried mesoporous carbon films were weighted and then soaked in an aqueous $\mathrm{H}_{2} \mathrm{SO}_{4} 1 \mathrm{M}$ solution for $18 \mathrm{~h}$. Two pieces of symmetrical carbon mesoporous films as electrodes, one piece of 110 mm-sized Whatman filter paper as a separator, and $\mathrm{H}_{2} \mathrm{SO}_{4} 1 \mathrm{M}$ as an electrolyte were designed. Stainlesssteel collectors were then sandwiched two films with a separator and placed in a two-electrode cell. Specific capacitance $\left(C_{s}\right)$ of the mesoporous carbon materials was calculated from cyclic voltammogram based on the equation $\left(C_{s}=2\left(I / V^{*} m\right)\right)\left(\mathrm{F} \cdot \mathrm{g}^{-1}\right)$, where $I(\mathrm{~A}), V(\mathrm{~V}), m(\mathrm{~g})$ are the current got from the charge scan of the CV curve, the voltage sweep rate, and the mass of the sample per electrode, respectively.

\section{Results and Discussion}

Alginic acid fibers were prepared from treating the brown seaweeds with sodium carbonate solution followed by precipitating in dilute hydrochloric acid with the extraction yield of $10 \%$. Fourier transform infrared (FTIR) spectrum (Figure 1(a)) of the extracted product shows O-H stretching band at $3370 \mathrm{~cm}^{-1}$, C-H stretching band at $2924 \mathrm{~cm}^{-1}$, C-O stretching band at $1716 \mathrm{~cm}^{-1}$, and O-C-H stretching band at $1040 \mathrm{~cm}^{-1}$ [28]. These strong absorption peaks in the carbonyl region are characteristic of alginic acid. Transmission electron microscopy (TEM) images (Figure 1(b)) of the dilute alginic acid solution (0.05 wt\%) show the diameters of $10-15$ $\mathrm{nm}$ and lengths of $1-2 \mu \mathrm{m}$ characteristic of individual nanofibers. The goal of preparing NAA films from the self-assembly of NAA is to template hierarchical mesoporous silica films and convert porous carbon supercapacitors, we set out to determine the best $\mathrm{pH}$ condition of the aqueous NAA hydrogels for the self-assembly of the pure solid films. We found that the NAA gels were dissolved in basic aqueous media $(\mathrm{pH} \geq 11)$ to form a sodium alginate solution. The self-assembly of the sodium alginate solution with silica sol-gel precursors was achieved to produce silica-sodium alginate composite films under drying at ambient conditions. However, the silica-sodium alginate composite films were melted out in the basic media under carbonization. The NAA solution mostly formed a wet hydrogel film under air-drying 
(a)
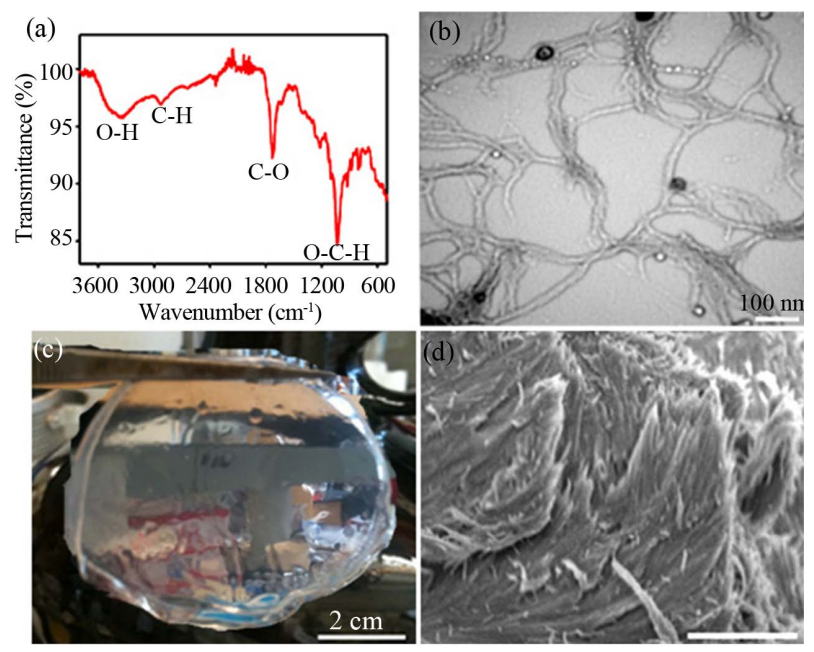

Figure 1. Nanofibrillar alginic acid extracted from brown seaweeds. (a) FTIR spectrum and (b) TEM image of the nanofibrillar alginic acid. (c) A photograph of a pure wet film prepared by assembling a nanofibrillar alginic acid suspension. (d) SEM image viewed along an edge of the pure nanofibrillar alginic acid film, scale bar $=100 \mu \mathrm{m}$.

when adjusting $\mathrm{pH}$ of the NAA solution to 4.5 by using dilute $\mathrm{HCl}$. However, the calcination and carbonization of the hydrogel composite films led to collapsed structures due to their strong volume shrinkage during annealing. A pure solid films can be formed from drying the aqueous NAA suspension at $\mathrm{pH} \sim 5-8$, however tetraalkoxysilane did not hydrolyze in this neutral medium to produce silica-NAA composites.

We found that the aqueous NAA gels became a stable suspension by adding an appropriate volume of acetic acid-sodium acetate buffer to decrease $\mathrm{pH}$ to 4.5 . The homogeneous suspension was dried to form a NAA solid film and silica-NAA composite films can be prepared at the $\mathrm{pH}$ of 4.5 . An aqueous NAA suspension was prepared at the concentration of $5.0 \mathrm{wt} \%$ and $\mathrm{pH} 4.5$ and used as precursor and liquid template for replicating the porous materials. The role of the acetic acid and sodium acetate buffer reduces hydrogen bonds of the alginic acid molecules in acidic media [29]. This allowed us to cast a pure NAA film by solidifying the aqueous suspension at ambient conditions within ca. $72 \mathrm{~h}$. Water was removed out of the film by soaking in anhydrous ethanol followed by vacuum-drying to obtain a crack-free solid film (Figure $1(\mathrm{c})$ ). Scanning electron microscopy (SEM) images show the morphology of a pure NAA film shown in Figure 1(d). In the image, the alginic acid nanofibers appear fairly oriented parallel to each other, which look more orderly than the individual nanofibers, demonstrating a self-assembled organization of the alginic acid nanofibers. The hierarchical parallel structure of the NAA films extracted from the brown seaweeds showed in our work has not previously been reported. We have thus used the hierarchical phase of the stable aqueous NAA suspension to template solid-state replicas.

We wonder whether self-assembling the aqueous alginic acid suspension with alkoxysilane may yield silica-surrounded alginic acid composites that serve as effective derivatives to produce porous silica and carbon films by calcination and carbonization, respectively. We thus set out to determine this concept namely, a silicaNAA composite suspension was prepared by mixing tetramethyl orthosilicate to the NAA suspension (5.0 wt $\%, \mathrm{pH} \sim 4.5$ ) a fixed silica/NAA proportion of 30:70 $\mathrm{wt} \%$ under stirring at room temperature for $1 \mathrm{~h}$ followed by adding $0.6 \mathrm{~mL}$ of concentrated ammonia. During the addition, $\mathrm{Si}\left(\mathrm{OCH}_{3}\right)_{4}$ underwent hydrolysis and condensation to produce a homogeneous suspension. After the reaction, films were cast, yielding a crack-free solid film of silica-NAA composites after drying. We found that the organization of the NAA template employed was transferred to the silica-NAA composites.

The morphological and structural characterizations of the silica-NAA composites were shown in Figure 2. Drying the composite suspensions gave large, crack-free silica/NAA composites films (Figure 2(a)) that show birefringence under POM (Figure 2(b)), proving that the presence of silica precursors did not significantly disturb the alignment of NAA. Aligned structures originating from the arrangement of NAA were observed by TEM of the composites (Figure 2(c)). These features may reflect a natural replication of the organic-inorganic composite films. In contrast to the pure NAA films, the composites could not be dispersed in water. FTIR spectrum (Figure 2(d)) of the silica/NAA composites shows IR bands associated with vibrational modes of NAA and silica, demonstrating the sol-gel condensation between silica and alginic acid components.

Mesoporous silica films were generated after removal of the NAA template by calcination of the composites at $550^{\circ} \mathrm{C}$ under air (Figure 3(a)). The pure silica films appear more transparent and thinner than the composite films, resulting from loss of the alginic acid by combustion. POM image (Figure 3(b)) of the pure silica films show birefringent texture, reflecting the formation of an aligned porous structure. The hierarchical organization of the pure silica films was evident in SEM images. A cracked film shows a relatively smooth top surface and, from the cross-section, the film is about $50 \mu \mathrm{m}$ thick (Figure 3(d)). Electron microscopy viewed at high magnification reveals that there are rod-shaped morphology of the films (Figure 3(e)). During templation of the mesoporous silica with NAA, the hierarchical organization of NAA was transferred to the silica and the nanometer-scale feature was maintained during calcination.

The structures of the silica-NAA composites and the mesoporous silica were also characterized by thermogra- 

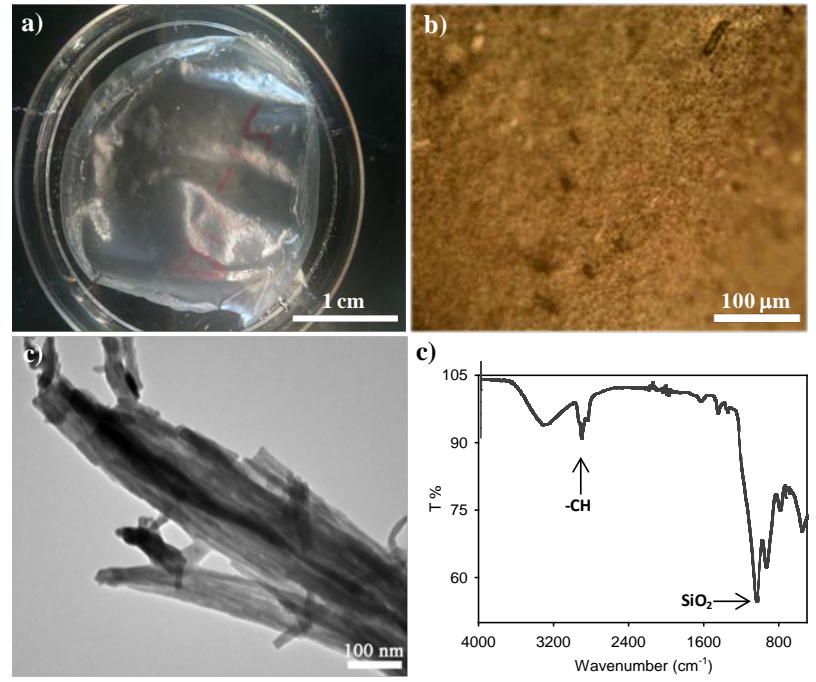

Figure 2. Evaporation-induced self-assembly of alkoxysilane with NAA to form silica/NAA composites. (a) Photograph of the silica/NAA composites showing transparency of the film. (b) POM image of the silica/NAA composites. (c) TEM image of the silica/NAA composites. (d) FTIR spectrum of the silica/NAA composites.
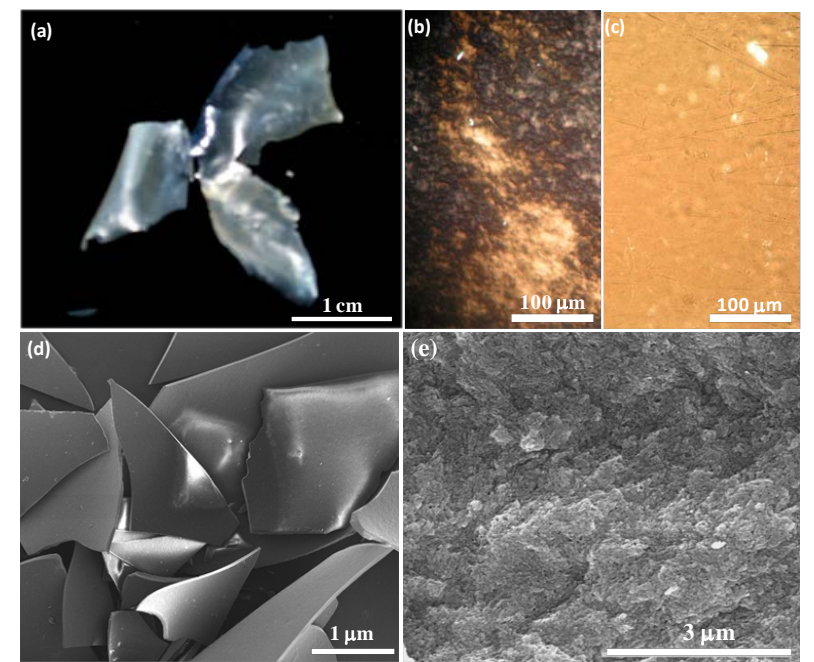

Figure 3. Hierarchical porous silica films after removal of the alginic acid template by calcincation. (a) Photograph of the silica films. (b) POM image of the silica films. (c) POM image of the silica films absorbed water, inset of waterabsorbed silica films. (d) SEM image of the calcined silica films at low magnification. (e) SEM image of the calcined silica films viewed along fracture cross-sections at high magnification.

vimetric analysis (TGA), powder X-ray diffraction (PXRD), and elemental analysis; the TGA curve and PXRD pattern are shown in Figure 4. TGA analysis (Figure 4(a)) shows that a total mass loss of the alginic acid in the composites was $\sim 70 \mathrm{wt} \%$ in the range of 100 to $500^{\circ} \mathrm{C}$. PXRD (Figure $4(\mathrm{~b})$ ) of the calcined silica obtained after alginic acid removal shows only a broad peakat $2 \theta \approx 22^{\circ}$ attributed to amorphous silica. The elemental analysis of the composites was found to have 40 wt\% carbon, while this component is absent in the calcined silica films. These results are consistent with the complete removal of NAA in the composites to yield the pure silica films.

Porosity of the silica films was determined by nitrogen adsorption-desorption studies. Figure 4(c) shows that the silica film reveals a typical type-IV isotherm with a hysteretic loop in the range of $0.6-1.0 \mathrm{p} / \mathrm{p}_{\mathrm{o}}$, which indicates the introduction of mesoporosity into the silica solid. Brunauer-Emmett-Teller (BET) surface area and pore volume were found to be $500 \mathrm{~m}^{2} \cdot \mathrm{g}^{-1}$ and $0.50 \mathrm{~cm}^{3} \cdot \mathrm{g}^{-1}$, respectively. Pore diameters determined by a BJH (Barrett-Joyner-Halenda) analysis are $\sim 8.0 \mathrm{~nm}$, consistent with the diameters of individual alginic acid nanofibers (10 - $15 \mathrm{~nm}$ ) after removal of NAA by heating. TEM images (Figure $4(d)$ ) of the silica films further confirmed the formation of an aligned porous network within the films, where the mesochannels run parallel to a preferred orientation over each layer. The pore networks were observed consistent with the hierarchical aligned organization of the mesoporous structure. The silica films with oriented mesochannels have a large structural anisotropy, which results in the appearance of the birefringent texture under POM. The hierarchical aligned organization and mesoporosity of the silica replicas was elucidated by solvent absorption into the film. As seen in inset of Figure 3(c), when a water droplet was placed on a film, the water was rapidly absorbed and immediately the film appeared transparent and colorless. Examination of this film under POM (Figure 3(c)) shows that the birefringence disappeared; after drying the film, it returned to its original state. This result shows that the water fills the pores of the silica. The combination of mesochannels and anisotropy in a glass film could make it useful in applications such as optical elements, catalyst supports, adsorbents, and solar cells. As well, a further research direction could be to use these materials as a hard template to replicate hierarchical orientation into other materials.

The composite films were carbonized under nitrogen at $1000^{\circ} \mathrm{C}$ and then etched the silica with dilute $\mathrm{NaOH}$ to generate mesoporous carbon with hierarchical pore structure. Silica-carbon composites appear black color and volume shrinkage with shape similar to the silicaNAA films (Figure 5(a)). The carbonization yield of the NAA-to-carbon conversion determined by thermogravimetric analysis (TGA) analysis (Figure 5(b)) was found to be $\sim 23 \%$. The removal of the silica generated the pure carbon films was determined by TGA. A nearcomplete burn of the carbon occurred at $500^{\circ} \mathrm{C}-750^{\circ} \mathrm{C}$ in air and remained a small residual ash of $\sim 2 \mathrm{wt} \%$. Powder X-ray diffraction (PXRD) pattern (Figure 5(c)) 

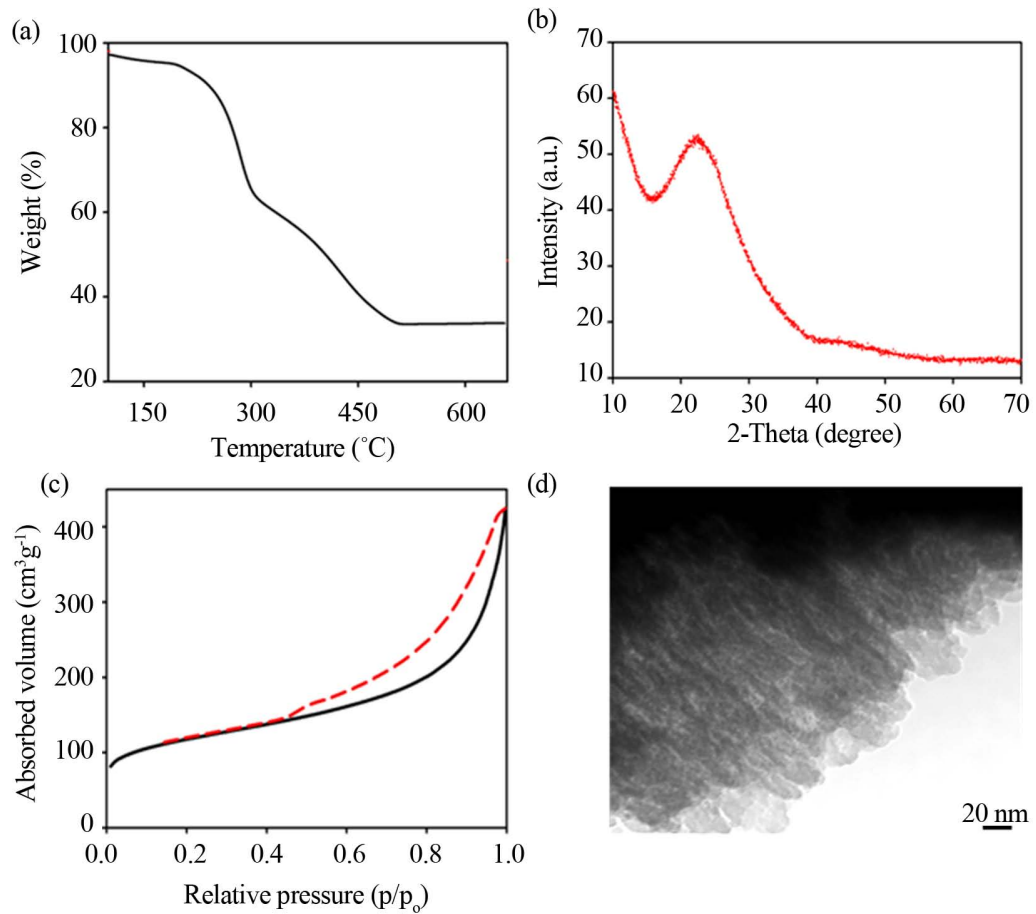

d)

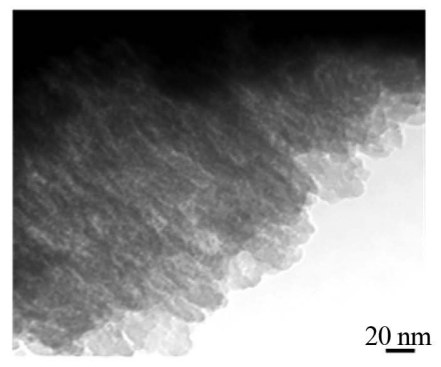

Figure 4. Structural characterization of the calcined silica films. (a) TGA analysis of the silica-NAA composites running under air. (b) PXRD pattern of the silica films. (c) Nitrogen adsorption/desorption isotherms of the silica films. (d) TEM image of the silica films.

(a)

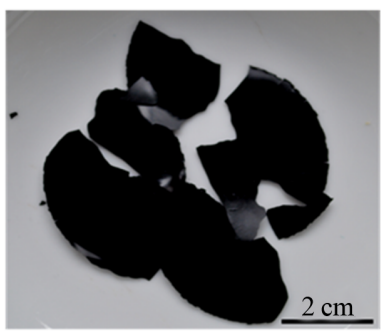

(c)

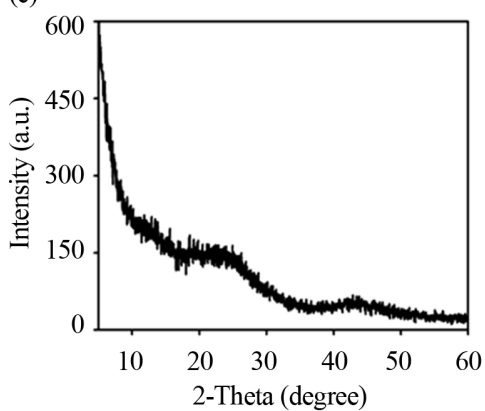

(b)

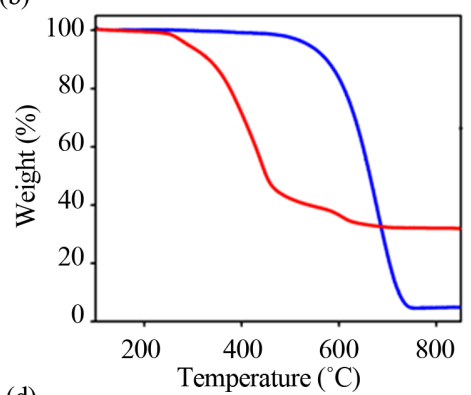

(d)

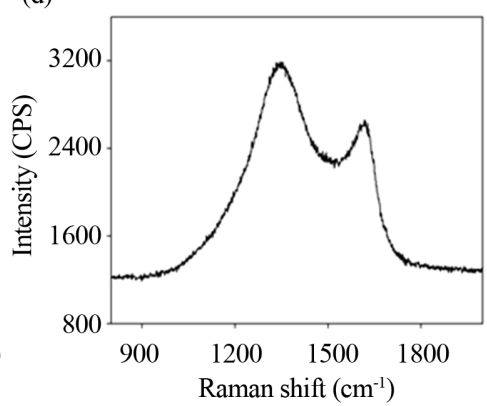

Figure 5. Structural characterization of the carbon films. (a) A photograph of carbon films prepared by carbonizing the composites and subsequent etching the silica. (b) TGA curves of silica-NAA composites and carbon films. (c) PXRD and (d) Raman spectra of the carbon materials.

of the carbon sample reveals two broad peaks at $23^{\circ}$ and $43^{\circ}$ attributed to turbostratic carbon [27]. Raman spectrum (Figure 5(d)) of the pure carbon shows two bands at $1350 \mathrm{~cm}^{-1}$ (D band) and $1580 \mathrm{~cm}^{-1}$ (G band) correspond- ing to amorphous carbon structure [27]. Elemental analysis of the pure carbon films was found to be $\sim 90$ wt $\%$ carbon and $~ 1.2 \mathrm{wt} \%$ hydrogen.

The porosity of the carbon materials was studied using 
nitrogen adsorption/desorption. Figure 6(a) shows the carbon films having a type-IV isotherm with type-H2 hysteresis loop characteristic of the mesoporous structure. This demonstrated the formation of mesopores in the carbon structure. The mesoporous carbon films have Brunauer-Emmett-Teller (BET) surface area of 913 $\mathrm{m}^{2} \cdot \mathrm{g}^{-1}$ and pore volume of $0.73 \mathrm{~cm}^{3} \cdot \mathrm{g}^{-1}$. In comparison with the alginic acid-derived carbon materials [27,30], our prepared mesoporous carbon films show a higher specific surface area because we took advantages of the sol-gel silica acted as an oriented pore agent for introducing mesoporosity into the carbon structure. TEM image (Figure 6(b)) of the mesoporous carbon further revealed the formation of an aligned porous network in the carbon films. The shape of the carbon rods was observed due mainly to the breaking of the starting alginic acid nanofibers during carbonization, while their dimensions resemble the alginic acid nanofibers. The hierarchical parallel organization of the mesoporous carbon materials was determined using SEM images. Top view of a surface perpendicular to the cross-section of the film shows that the rod-shaped carbons with a visible roughening of the surface was arranged in parallel arrays with elongated rods separated by holes (Figure 6(c)).

The electrochemical property for mesoporous carbon supercapacitor was studied by using cyclic voltam-

a)

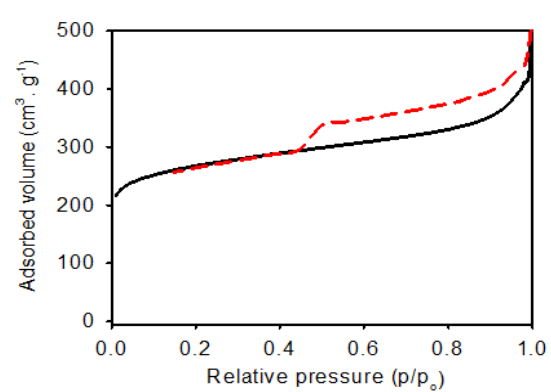

c)

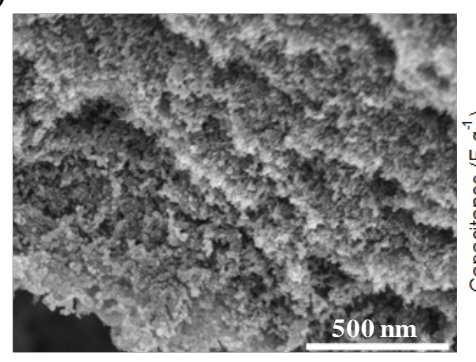

mogram (CV) technique. Symmetrical cells with a two-electrode configuration of two mesoporous carbon electrodes were constructed and acted in $1 \mathrm{M} \mathrm{H}_{2} \mathrm{SO}_{4}$ electrolyte. Figure 6(d) shows the electrochemical performance of the mesoporous carbon over a broad range of the scan rates. CV curves of the mesoporous carbon keep a rectangular shape at a low scan rate of $5 \mathrm{mV} \cdot \mathrm{s}^{-1}$ and a high scan rate of $100 \mathrm{mV} \cdot \mathrm{s}^{-1}$. The CV curve mostly remains the leaf-like shape even at an fast scan rate of $100 \mathrm{mV} \cdot \mathrm{s}^{-1}$. The specific capacitance $\left(C_{s}\right)$ of the mesoporous carbon calculated from the $\mathrm{CV}$ charge curve at $5 \mathrm{mV} \cdot \mathrm{s}^{-1}$ is $276 \mathrm{~F} \cdot \mathrm{g}^{-1}$ in the aqueous $\mathrm{H}_{2} \mathrm{SO}_{4} 1 \mathrm{M}$ solution, which is higher than the value reported for the porous carbon prepared from crude seaweeds under analogous conditions [31]. The highly electrochemical cycling stability of the mesoporous carbon materials could arise from their high specific surface area and aligned porous network. Moreover our materials are in a film form and are thus used for supercapacitor without binders required. The capacitance retained with strongly increasing frequency implying that the mesoporous carbon exhibited both superior electrical conductivity and highly charge storage.

\section{Conclusion}

In conclusion, hierarchical porous silica and carbon films

b)

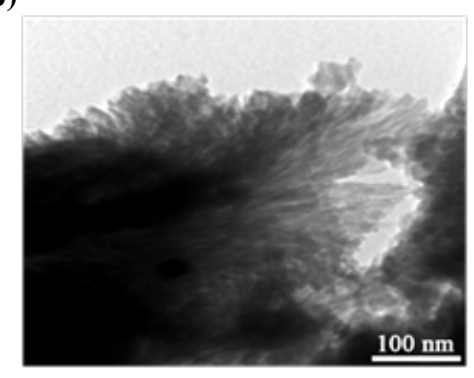

d)

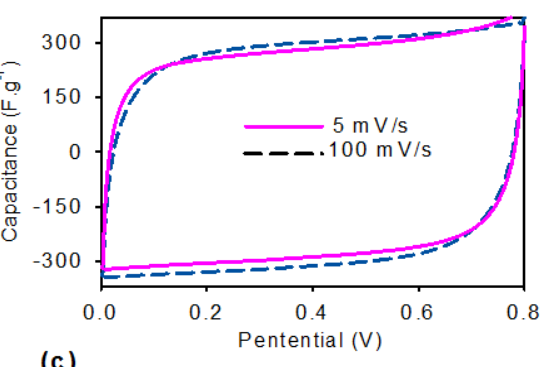

(c)

Figure 6. Porosity and electrochemical property of the carbon films. (a) Nitrogen adsorption/desorption isotherms of mesoporous carbon films. (b) TEM image of the mesoporous carbon. (c) SEM image of the mesoporous carbon. (d) Cyclic voltammogram (CV) curves of the mesoporous carbon films in aqueous $\mathrm{H}_{2} \mathrm{SO}_{4} 1 \mathrm{M}$ in a broad range of scan rate from 5 to $100 \mathrm{mV} \cdot \mathrm{s}^{-1}$. 
have been synthesized by polymeric liquid templating with nanofibrillar alginic acid assemblies. The brown seaweeds were extracted with sodium carbonate solution followed by precipitating in dilute hydrochloric acid to produce the nanofibrillar alginic acid. We used an acetic acid-sodium acetate buffer to adjust $\mathrm{pH}$ of the alginic acid hydrogels and formed a stable aqueous nanofibrillar alginic acid suspension with the concentration of $5.0 \mathrm{wt} \%$ and $\mathrm{pH} \sim 4.5$. The hierarchical phase of the nanofibrillar alginic acid was used to template crack-free silica-alginic acid composite films through evaporation-induced selfassembly of alkoxysilane with nanofibrillar alginic acid. After calcination of the alginic acid template, silica replicas afforded that showed mesoporosity and birefringence arising from a transfer of hierarchical aligned organization of the nanofibrillar alginic acid into the silica solid. Conversion of the silica-alignic acid composites to silica-carbon composites by carbonization and subsequent etching the silica recovered hierarchical mesoporous carbon supercapacitors. This versatile template-directed approach allows for the large-scale preparation of these novel materials from widely available biomass resources.

\section{Acknowledgments}

This work was supported by the National Foundation for Science and Technology development of VietnamNAFOSTED No. 104.03-2012.54.

\section{REFERENCES}

[1] C. Sanchez, H. Arribart and M. M. Giraud Guille, "Biomimetism and Bioinspiration as Tools for the Design of Innovative Materials and Systems," Nature Materials, Vol. 4, No. 4, 2005, pp. 277-288. http://dx.doi.org/10.1038/nmat1339

[2] J. Aizenberg, J. C. Weaver, M. S. Thanawala1, V. C. Sundar, D. E. Morse and P. Fratzl, "Skeleton of Euplectella sp.: Structural Hierarchy from the Nanoscale to the Macroscale,” Science, Vol. 309, No. 5732, 2005, pp. 275-278. http://dx.doi.org/10.1126/science.1112255

[3] W.-J. Chung, J.-W. Oh, K. Kwak, B. Y. Lee, J. Meyer, E. Wang, A. Hexemer and S.-W. Lee, "Biomimetic Self-templating Supramolecular Structures,” Nature, Vol. 478, No. 7369, 2011, pp. 364-368. http://dx.doi.org/10.1038/nature10513

[4] H. Zhou, T. Fan and D. Zhang, "Biotemplated Materials for Sustainable Energy and Environment: Current Status and Challenges," ChemSusChem, Vol. 4, No. 10, 2011, pp. 1344-1387. http://dx.doi.org/10.1002/cssc.201100048

[5] V. Percec, "Bioinspired Supramolecular Liquid Crystals. Philosophical Transactions of the Royal Society A: Mathematical,” Physical and Engineering Sciences, Vol. 364, No. 1847, 2006, pp. 2709-2719.

[6] S. H. Tolbert, "Magnetic Field Alignment of Ordered Si-
licate-Surfactant Composites and Mesoporous Silica," Science, Vol. 278, No. 5336, 1997. pp. 264-268. http://dx.doi.org/10.1126/science.278.5336.264

[7] P. Feng, X. Bu and D. J. Pine, "Control of Pore Sizes in Mesoporous Silica Templated by Liquid Crystals in Block Copolymer-Cosurfactant-Water Systems,” Langmuir, Vol. 16, No. 12, 2000. pp. 5304-5310. http://dx.doi.org/10.1021/la991444f

[8] H. Yang, N. Coombs, I. Sokolov and G. A. Ozin, "FreeStanding and Oriented Mesoporous Silica Films Grown at the Air-Water Interface,” Nature, Vol. 381, No. 6583, 1996, pp. 589-592.

http://dx.doi.org/10.1038/381589a0

[9] G. S. Attard, J. C. Glyde and C. G. Goltner, "Liquid-Crystalline Phases as Templates for the Synthesis of Mesoporous Silica,” Nature, Vol. 378, No. 6555, 1995, pp. 366368. http://dx.doi.org/10.1038/378366a0

[10] I. Hodgkinson and Q. H. Wu, "Inorganic Chiral Optical Materials,” Advanced Materials, Vol. 13, No. 12-13, 2001, pp. 889-897.

http://dx.doi.org/10.1002/1521-4095(200107)13:12/13<8 89::AID-ADMA889>3.0.CO;2-K

[11] B. F. G. Johnson, et al., "Superior Performance of a Chiral Catalyst Confined within Mesoporous Silica,” Chemical Communications, No. 13, 1999, pp. 1167-1168. http://dx.doi.org/10.1039/a902441g

[12] K. Kosuge, S. Kubo, N. Kikukawa and M. Takemori, "Effect of Pore Structure in Mesoporous Silicas on VOC Dynamic Adsorption/Desorption Performance,” Langmuir, Vol. 23, No. 6, 2007, pp. 3095-3102. http://dx.doi.org/10.1021/la062616t

[13] A. I. Hochbaum and P. Yang, "Semiconductor Nanowires for Energy Conversion,” Chemical Reviews, Vol. 110, No. 1, 2009, pp. 527-546. http://dx.doi.org/10.1021/cr900075v

[14] S. J.Woltman, G. D. Jay and G. P. Crawford, "LiquidCrystal Materials Find a New Order in Biomedical Applications,” Nature Materials, Vol. 6, No. 12, 2007, pp. 929-938. http://dx.doi.org/10.1038/nmat2010

[15] A. D. Augst, H. J. Kong and D. J. Mooney, “Alginate Hydrogels as Biomaterials,” Macromolecular Bioscience, Vol. 6, No. 8, 2006, pp. 623-633. http://dx.doi.org/10.1002/mabi.200600069

[16] A. Ikeda, A. Takemura and H. Ono, "Preparation of LowMolecular Weight Alginic Acid by Acid Hydrolysis,” Carbohydrate Polymers, Vol. 42, No. 4, 2000, pp. 421-425. http://dx.doi.org/10.1016/S0144-8617(99)00183-6

[17] E. Dujardin, M. Blaseby and S. Mann, "Synthesis of Mesoporous Silica by Sol-Gel Mineralisation of Cellulose Nanorod Nematic Suspensions,” Journal of Materials Chemistry, Vol. 13, No. 4, 2003, pp. 696-699. http://dx.doi.org/10.1039/b212689c

[18] B. Alonso and E. Belamie, "Chitin-Silica Nanocomposites by Self-Assembly," Angewandte Chemie International Edition, Vol. 49, No. 44, 2010, pp. 8201-8204. http://dx.doi.org/10.1002/anie.201002104 
[19] E. Belamie, M. Y. Boltoeva, K. Yang, T. Cacciaguerra and B. Alonso, "Tunable Hierarchical Porosity from SelfAssembled Chitin-Silica Nano-Composites,” Journal of Materials Chemistry, Vol. 21, No. 42, 2011, pp. 1699717006. http://dx.doi.org/10.1039/c1jm12110c

[20] W. Ogasawara, W. Shenton, S. A. Davis and S. Mann, "Template Mineralization of Ordered Macroporous Chitin-Silica Composites Using a Cuttlebone-Derived Organic Matrix," Chemistry of Materials, Vol. 12, No. 10, 2000, pp. 2835-2837. http://dx.doi.org/10.1021/cm0004376

[21] K. Spinde, M. Kammer, K. Freyer, H. Ehrlich, J. N. Vournakis and K. Brunner, "Biomimetic Silicification of Fibrous Chitin from Diatoms,” Chemistry of Materials, Vol. 23, No. 11, 2011, pp. 2973-2978. http://dx.doi.org/10.1021/cm200677d

[22] A. Stein, Z. Wang and M. A. Fierke, "Functionalization of Porous Carbon Materials with Designed Pore Architecture,” Advanced Materials, Vol. 21, No. 3, 2009, pp. 265293. http://dx.doi.org/10.1002/adma.200801492

[23] C. Z. Yuan, B. Gao, L. F. Shen, S. D. Yang, L. Hao, X. J. Lu, F. Zhang, L. J. Zhanga and X. G. Zhang, "Hierarchically Structured Carbon-Based Composites: Design, Synthesis and Their Application in Electrochemical Capacitors,” Nanoscale, Vol. 3, No. 2, 2011, pp. 529-545. http://dx.doi.org/10.1039/c0nr00423e

[24] J. Hyun Young, et al., "Transparent, Flexible Supercapacitors from Nano-Engineered Carbon Films," Scientific Reports, Vol. 2, 2012.

[25] A. Ghosh and Y. H. Lee, "Carbon-Based Electrochemical Capacitors,” ChemSusChem, Vol. 5, No. 3, 2012, pp. 480-
499. http://dx.doi.org/10.1002/cssc.201100645

[26] R. J. White, V. Budarin, R. Luque, J. H. Clark and D. J. Macquarriea, "Tuneable Porous Carbonaceous Materials from Renewable Resources,” Chemical Society Reviews, Vol. 38, No. 12, 2009, pp. 3401-3418. http://dx.doi.org/10.1039/b822668g

[27] X.-L.Wu, L.-L. Chen, S. Xin, Y.-X. Yin, Y.-G. Guo, Q.-S. Kong and Y.-Z. Xia, "Preparation and Li Storage Properties of Hierarchical Porous Carbon Fibers Derived from Alginic Acid,” ChemSusChem, Vol. 3, No. 6, 2010, pp. 703-707. http://dx.doi.org/10.1002/cssc.201000035

[28] C. Jeon, J. Y. Park and Y. J. Yoo, "Characteristics of Metal Removal Using Carboxylated Alginic Acid,” Water Research, Vol. 36, No. 7, 2002, pp. 1814-1824. http://dx.doi.org/10.1016/S0043-1354(01)00389-X

[29] C. Tapia, V. Montezuma and M. Yazdani-Pedram, "Microencapsulation by Spray Coagulation of Diltiazem $\mathrm{HCl}$ in Calcium Alginate-Coated Chitosan,” AAPS PharmSciTech, Vol. 9, No. 4, 2008, pp. 1198-1206. http://dx.doi.org/10.1208/s12249-008-9164-3

[30] R. J. White, C. Antonio, V. L. Budarin, E. Bergstrom, J. Thomas-Oates and J. H. Clark, "Polysaccharide-Derived Carbons for Polar Analyte Separations,” Advanced Functional Materials, Vol. 20, No. 11, 2010, pp. 1834-1841. http://dx.doi.org/10.1002/adfm.201000169

[31] E. Raymundo-Piñero, M. Cadek and F. Béguin, “Tuning Carbon Materials for Supercapacitors by Direct Pyrolysis of Seaweeds," Advanced Functional Materials, Vol. 19, No. 7, 2009, pp. 1032-1039. http://dx.doi.org/10.1002/adfm.200801057 